and early phase of the progression of ALS-like disease in mice. By contrast, expression of mutant SOD1 in microglia was found to be a primary factor in later disease progression.

The authors conclude that the initiation and progression of ALS are distinct phases involving different cell types; mutant SOD1 expression in motor neurons underlies the initiation of ALS, whereas disease progression is linked with mutant SOD1 expression in microglia. These findings have important implications for the development of therapies for the treatment of ALS.

Original article Boillée S et al. (2006) Onset and progression in inherited ALS determined by motor neurons and microglia. Science 312: 1389-1392

\section{Increased common carotid artery stiffness is associated with ischemic stroke}

Increased common carotid artery (CCA) stiffness is an independent predictor of cardiovascular mortality in end-stage renal disease, but little is known about its relationship with cerebrovascular disease. Tsivgoulis et al. have therefore investigated the relationship between CCA stiffness-determined by high-resolution B-mode ultrasonography - and ischemic stroke.

The study included 193 patients who were admitted to an acute stroke unit in Greece with a first-ever ischemic stroke, and 106 agematched and sex-matched controls (who had been referred to the hypertension center of the same institution).

CCA distensibility was significantly lower (i.e. stiffness was greater) in stroke patients than in control subjects $(P=0.005)$; this finding remained true after adjustment for known stroke risk factors such as blood pressure, height and diastolic CCA diameter $(P=0.007)$. CCA intimamedia thickness (IMT) values were significantly higher in patients with ischemic stroke than in control patients $(P<0.001)$. In multivariate analyses, CCA-IMT and CCA distensibility were the only statistically significant predictors of ischemic stroke: each $1 \mathrm{SD}$ increase in CCA-IMT increased the risk of stroke by $167.0 \%$, and each 1 SD decrease in CCA distensibility increased the risk of stroke by $59.0 \%$.

The authors conclude that increased CCA stiffness is associated with ischemic stroke, even after adjustment for established stroke risk factors and CCA-IMT, a marker of generalized atherosclerosis. Noninvasive measurements of CCA stiffness might, therefore, be useful for predicting stroke risk independently of the extent of atherosclerosis, but further studies are needed.

Original article Tsivgoulis G et al. (2006) Common carotid arterial stiffness and the risk of ischaemic stroke. Eur J Neurol 13: $475-481$

\section{Caudal zona incerta stimulation improves contralateral parkinsonism}

Deep brain stimulation is commonly used in the treatment of Parkinson's disease (PD). Although the subthalamic nucleus (STN) is the most usual target for stimulation, controversy exists over whether this is the optimum location. Plaha et al. now report high-frequency stimulation of the caudal part of the zona incerta nucleus (cZl) to be the most effective.

Between April 2002 and June 2004, 64 patients with medically refractory idiopathic PD underwent deep brain stimulation surgery, using a novel MRI-directed method of lead implantation; 17 patients had the best therapeutic contact in the postero-dorsal STN, 20 dorsomedial/medial to the STN (d/mSTN), and 27 posterior to the STN within the cZI. Patients were evaluated a median of 6 months after surgery. Following stimulation, the cZl group showed the most marked reductions in Unified Parkinson's Disease Rating Scale III motor score and adjusted 'off-on' rigidity score, as well as the greatest improvements in tremor and bradykinesia. The differences in dyskinesia scores, levodopa medication reduction and stimulation parameters were not statistically different between the groups; however, four patients in the $\mathrm{d} / \mathrm{mSTN}$ group experienced adverse effects, and bilateral stimulation in these patients led to slurred speech and balance difficulties.

These results indicate that abnormal zona incerta activity could have an important role in PD. The data need replicating in larger randomized, controlled studies, but offer a potential improvement for the surgical management of PD.

Original article Plaha P et al. (2006) Stimulation of the caudal zona incerta is superior to stimulation of the subthalamic nucleus in improving contralateral parkinsonism. Brain 129: 1732-1747 